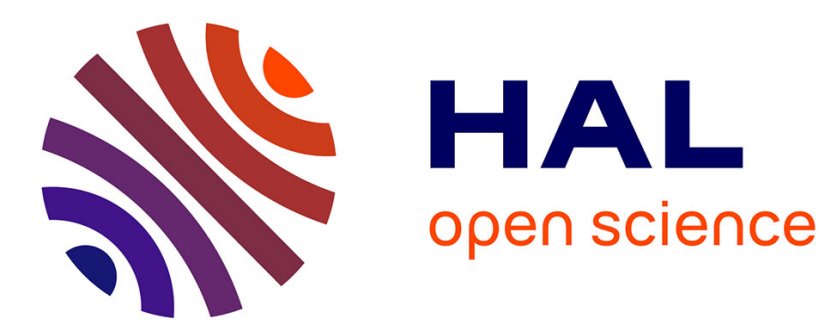

\title{
Component ranking by Birnbaum importance in presence of epistemic uncertainty in failure event probabilities
}

Piero Baraldi, Michele Compare, Enrico Zio

\section{- To cite this version:}

Piero Baraldi, Michele Compare, Enrico Zio. Component ranking by Birnbaum importance in presence of epistemic uncertainty in failure event probabilities. IEEE Transactions on Reliability, 2013, 62 (1), pp.37-48. 10.1109/TR.2013.2240885 . hal-00926352

\section{HAL Id: hal-00926352 \\ https://hal-centralesupelec.archives-ouvertes.fr/hal-00926352}

Submitted on 9 Jan 2014

HAL is a multi-disciplinary open access archive for the deposit and dissemination of scientific research documents, whether they are published or not. The documents may come from teaching and research institutions in France or abroad, or from public or private research centers.
L'archive ouverte pluridisciplinaire HAL, est destinée au dépôt et à la diffusion de documents scientifiques de niveau recherche, publiés ou non, émanant des établissements d'enseignement et de recherche français ou étrangers, des laboratoires publics ou privés. 


\title{
Component ranking by Birnbaum importance in presence of epistemic uncertainty in failure event probabilities
}

\author{
P. Baraldi ${ }^{1}$, M. Compare ${ }^{1}$, E. Zio ${ }^{1,2, *}$, senior member, IEEE \\ ${ }^{1}$ Politecnico di Milano, Italy \\ ${ }^{2}$ Chair on Systems Science and the Energetic Challenge, European Foundation for New Energy-Electricité de \\ France, Ecole Centrale Paris and Supelec, France \\ *Corresponding author: enrico.zio@polimi.it, enrico.zio@ecp.fr, enrico.zio@supelec.fr
}

Index Terms - Birnbaum importance measure, possibility theory, importance ranking.

\begin{abstract}
Birnbaum Importance Measure (IM) allows ranking the components of a system with respect to the impact that their failures have on the system's performance, e.g., its reliability or availability. Such ranking is done in industry to efficiently manage Operation and Maintenance (O\&M) activities, and to optimize plant design. In the computation of the Birnbaum IM of the components, uncertainty in the parameters of the system model is often neglected. This neglect may lead to erroneous, possibly nonconservative ranking. In this work, we develop a method based on Possibility Theory (PT) for giving due account to epistemic uncertainties in Birnbaum IMs. An example is given with reference to the components of the Auxiliary FeedWater System (AFWS) of a Nuclear Power Plant (NPP).
\end{abstract}

\section{Acronyms}

$\begin{array}{ll}\text { AFWS } & \text { Auxiliary FeedWater System } \\ \text { CDF } & \text { Cumulative Distribution Function } \\ \text { DSTE } & \text { Dempster-Shafer Theory of Evidence } \\ \text { IM } & \text { Importance Measure } \\ \text { NPP } & \text { Nuclear Power Plant } \\ \text { O\&M } & \text { Operation and Maintenance } \\ \text { PT } & \text { Possibility Theory }\end{array}$




$\begin{array}{ll}\text { PWR } & \text { Pressurized Water Reactor } \\ \text { RBD } & \text { Reliability Block Diagram } \\ \text { UIM } & \text { Uncertainty Importance Measure } \\ \text { UoD } & \text { Universe of Discourse }\end{array}$

\section{Notation}

$n$

$Q_{j}$

Q

$q_{j}$

$f_{Q_{j}}\left(q_{j}\right) \quad$ Probability density function (pdf) of the variable $Q_{j}$

$F_{Q_{j}}\left(q_{j}\right)$

$N_{Q_{j}}(A)$

$\Pi_{Q_{j}}(A)$

$\pi_{Q_{j}}\left(q_{j}\right)$

$I_{j}^{B}$

$\mathbf{I}^{B}$

$g_{j}(\square)$

$U(t)$

Failure probability associated to the $j$-th component

Vector containing the variables $\left(Q_{1}, \ldots, Q_{n}\right)$

The generic value taken by $Q_{j}$

CDF of the variable $Q_{j}$

Possibility function of the variable $Q_{j}$

Birnbaum IM of the $j$-th component

Vector containing the components' IMs $\left(I_{1}^{B}, \ldots, I_{n}^{B}\right)$

System failure probability at time $t$
Number of components in the system

Necessity measure associated to the subset A of the domain of the variable $Q_{j}$

Possibility measure associated to the subset $A$ of the domain of the variable $Q_{j}$

Function linking the components failure probabilities $\mathbf{Q}$ to the Birnbaum IM $I_{j}^{B}$

\section{Introduction}

The notion of risk involves some kind of loss or damage that might be received by a target, and the uncertainty of its transformation in an actual loss or damage [1]. A relevant outcome of a risk analysis of a complex industrial system is the quantification of the importance of the component failure events with respect to the system overall risk. To this aim, various Importance Measures (IMs) have been proposed [2]- 
[16]. The importance of a component failure depends on its role in the failure logic structure of the system and its probability of occurrence, which captures the (aleatory) uncertainty due to the inherent variability of the failure behaviors. In practice, IMs are useful because they allow one to compare the importance of the different component (random) failure events.

The IM considered in this work is the Birnbaum IM, which was originally introduced to assess the contributions to the system failure probability due to the failure probabilities of the various system components. In particular, components for which a variation in this probability results in the largest variation of the system reliability have the highest importance [2], [8]. Then, Birnbaum IM allows ranking the components of a system on the basis of the influence of their stochastic behaviors on the system reliability performance. Nowadays, it is widely used in industry for different purposes such as rationalizing or optimizing O\&M [9]- [12], performing Probabilistic Risk Assessment studies (e.g., in the nuclear field) [13] even when failure events are not $s$-independent [14] or the system is not coherent [15], addressing the component assignment problem [16], etc.

In the computation of IM, it is generally assumed that the values of the parameters governing the probabilistic distributions of the failure event occurrences are perfectly known (e.g., the failure rates). However, imprecise knowledge of these values is typically encountered in practical applications. This uncertainty is usually referred to as epistemic or reducible uncertainty [17].

In [18] and [19], it has been shown that disregarding the epistemic uncertainties in the component failure probabilities can lead to biased IMs values and ranking. To quantify the effect of epistemic uncertainties in IMs, Uncertainty IMs (UIMs) have been propounded [20]-[22]. According to [22], the main uncertainty contributors (i.e., with highest UIMs) tend to be different from the main risk contributors (i.e., with highest IMs). Thus, UIMs alone do not allow tackling the issue of ranking the importance of the components accounting for the epistemic uncertainty affecting their behaviors. In this respect, Aven and Nøkland have proposed a ranking method based on the couple (IM, UIM) [20].

A different approach to rank component IMs taking into account the epistemic uncertainty in the failure probabilities of the components has been proposed in [18], and applied to an industrial case study in [13]. 
This is a global sensitivity-based method based on a probabilistic exceedance measure that enables comparing the importance of two components. This method has been further investigated in [19], where the authors have used the Quicksort algorithm [23] for effectively ranking the component IMs.

These approaches handle epistemic uncertainty within the probability theory framework. Recently, resorting to probability distributions to represent insufficient knowledge has been questioned in risk assessment [24] because it forces assumptions which may not be justified by the available information, and may lose generality of the results. For example, ignoring whether a value of a parameter is more or less probable than any other value within a given range does not justify assuming a uniform probability distribution, which is the less informative probability distribution according to both the Laplace principle of insufficient reason and the maximum entropy criterion [24].

Recently, set-valued representations have been used to represent the epistemic uncertainty in situations where all that is known is that the parameter value belongs to a certain range [25]. Techniques based on Dempster-Shafer Theory of Evidence (DSTE), PT, Interval Analysis, and Fuzzy Set Theory have been proposed to represent the epistemic uncertainties associated to this type of information, in a way less committed to assumptions than that offered by the probability theory framework (e.g., see [25]-[38] for surveys and comparisons of these techniques).

In this context, the objective of this work is to propose a procedure for ranking the component IM in the case in which the epistemic uncertainty in the parameters of the component failure models is described using PT. This procedure is applied to a case study concerning the AFWS of a nuclear pressurized water reactor.

The presentation of the work is organized as follows. In Section 2, the Birnbaum IM and its calculation are recalled, the problem of uncertainty is stated, and a simple example of reference is worked out within the probability theory framework. The same case study is re-considered in Section 3 within the PT framework of representation. The AFWS case study is worked out in Section 4. Concluding remarks on the findings of the paper are provided in Section 5. 


\section{Uncertainty in IMs}

Consider a system made up of $n$ components, assumed binary (i.e., they have only two possible states 'working' and 'failed'), and s-independent.

The Birnbaum IM $I_{j}^{B}(t)$ of the $j$-th component of such system at time $t$ is given by [20]:

$I_{j}^{B}(t)=\frac{\partial U(\mathbf{Q}(t))}{\partial Q_{j}(t)}$

Simple analytical manipulation yields [2]

$I_{j}^{B}(t)=U_{j}^{+}(t)-U_{j}^{-}(t)$

- $U_{j}^{+}(t)=U\left(Q_{j}=1, \mathbf{Q}(t)\right)$ is the system failure probability when component $j$ is in failed state. It represents the maximum risk achievement if component $j$ is considered failed [2].

- $U_{j}^{-}(t)=U\left(Q_{j}=0, \mathbf{Q}(t)\right)$ is the system failure probability when component $j$ is functioning. It represents the maximum reduction in risk if component $j$ is considered working [2].

Consider, as an example, the system in Fig. 1, and assume to know the exact values of the component unreliabilities, $Q_{A}, Q_{B}, Q_{C}$. The system unreliability is

$U\left(Q_{A}, Q_{B}, Q_{C}\right)=Q_{A} Q_{B}+Q_{C}-Q_{A} Q_{B} Q_{C}$

The Birnbaum IMs of the components are then obtained by using (1) as

$\mathbf{I}^{B}=\left[\begin{array}{c}I_{A}^{B} \\ I_{B}^{B} \\ I_{C}^{B}\end{array}\right]=\left[\begin{array}{c}Q_{B}-Q_{B} Q_{C} \\ Q_{A}-Q_{A} Q_{C} \\ 1-Q_{A} Q_{B}\end{array}\right]$ 


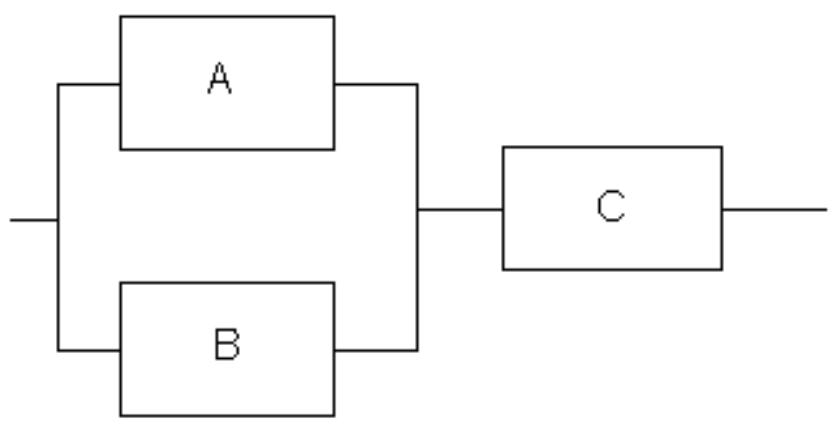

Fig. 1. System Reliability Block Diagram.

However, the exact values of the component failure probabilities $\mathbf{Q}(t)=\left(Q_{1}(t), Q_{2}(t), \ldots, Q_{n}(t)\right)$ are often not precisely known in practical situations, e.g., because they are assigned by an expert, or estimated through statistical means. Then, our objective is to rank the components according to their Birnbaum IMs, taking into account the epistemic uncertainties in the component failure probabilities. In general, whichever is the framework adopted to represent the epistemic uncertainties, this entails three successive steps:

1. representation the uncertainties on the component's failure probabilities,

2. propagation these uncertainties onto the component's IM values $I_{1}^{B}, \ldots, I_{n}^{B}$, and

3. ranking the component's IMs $I_{1}^{B}, \ldots, I_{n}^{B}$ taking into account the uncertainties obtained in the previous step.

Table I

Component unreliabilities, and IMs (taken from [19]).

\begin{tabular}{|c|c|c|}
\hline & $\begin{array}{c}\text { Unreliability: } \\
\text { Mean }\end{array}$ & $\begin{array}{c}\text { Unreliability: } \\
\text { Standard Deviation }\end{array}$ \\
\hline$A$ & 0.015 & 0.005 \\
\hline$B$ & 0.010 & 0.005 \\
\hline$C$ & 0.095 & 0.044 \\
\hline
\end{tabular}


In the remaining part of this section, we will consider the example in Fig. 1 assuming that the uncertain unreliabilities $Q_{j}, j=A, B, C$, are described by the Cumulative Distribution Functions $(\mathrm{CDFs}), F_{Q_{j}}\left(q_{j}\right)$, whose mean and standard deviations are reported in Table I. Notice that these distributions have been numerically obtained in [19] by assuming that an expert is able to represent the uncertainties in the component failure rates using lognormal distributions, and by propagating these uncertainties onto the component unreliabilities.

Fig. 2 (continuous line) shows the Birnbaum IMs distributions $F_{I_{j}^{B}}\left(i_{j}^{B}\right)$ obtained by propagating the uncertainties in the component unreliabilities $Q_{j}$ onto the Birnbaum IMs $I_{j}^{B}, j=A, B, C$.

\subsection{Components importance ranking, under a probabilistic representation of}

\section{uncertainty}

Here we recall the procedure propounded in [19] to rank the components according to their Birnbaum importance considering the probabilistic uncertainty in their IMs.

Let us consider two generic components $k$ and $s$; to establish which is the most important, the distribution of the random variable $\Delta_{k s}=\Delta_{k s}\left(I_{k}^{B}, I_{s}^{B}\right)=I_{k}^{B}-I_{s}^{B}$ is considered. Then, we compute the probability that $I_{k}^{B}$ is larger than $I_{s}^{B}$, referred to as 'exceedance measure' and given by $r_{k s}=1-F_{\Delta_{k s}}(0)$. Finally, the relation order between components $k$ and $s$ is obtained by comparing $r_{k s}$ to a threshold range $\left[T_{l}, 1-T_{l}\right]$, symmetric around 0.5 , and considering the following criteria.

- If $r_{k s} \geq 1-T_{l}$ then $k$ is more important than $s$.

- If $r_{k s} \leq T_{l}$ then $s$ is more important than $k$.

- If $T_{l} \leq r_{k s} \leq 1-T_{l}$ then $k$ is equally important to $s$. In this case, different kinds of additional constraints or targets should guide the ranking order (costs, repair times, failure impacts on public opinion, etc.).

In practice, the attention is concentrated on component $s$ if the decision maker judges 'large' enough (e.g., $>0.7$ ) the probability that component $s$ is more important than component $k$. 
Notice that there may be some cases in which the comparison of the Birnbaum importance of three generic components, $j, k, s$, may lead to a contradictory ranking which does not obey the transitive property, i.e., $I_{j}^{B}>I_{k}^{B}$ and $I_{k}^{B}>I_{s}^{B}$, but $I_{s}^{B}>I_{j}^{B}$. However, the demonstration in the Appendix shows that by setting $T_{l}$ lower than $1 / 3$, this contradictory ranking is avoided, and at worst it can happen that $I_{j}^{B}>I_{k}^{B}, I_{k}^{B}>I_{s}^{B}$, and $I_{s}^{B}=I_{j}^{B}$, which can be interpreted as a condition in which the three components are equally important.

Fig. 3 shows the CDF of the random variables $\Delta_{s k}$, with $s, k=A, B, C$ obtained by applying the probabilistic ranking procedure to the case study of Fig. 1. Because $r_{A C}=1-F_{\Delta_{A C}}(0)=P\left(I_{A}^{B}>I_{C}^{B}\right)=0$, and $r_{C B}=1-F_{\Delta_{C B}}(0)=P\left(I_{C}^{B}>I_{B}^{B}\right)=1$, one can conclude that component $C$ is certainty more important than components $A$ and $B$. With respect to the comparison between components $A$ and $B$ being the exceedance measure $r_{A B}=1-F_{\triangle_{A B}}(0)=0.23$, the ranking of the two components depends from the threshold ranges $\left[T_{l}, 1-T_{l}\right]$. Assuming, for example, that $T_{l}=0.3$, component $B$ turns out to be more important than component A.
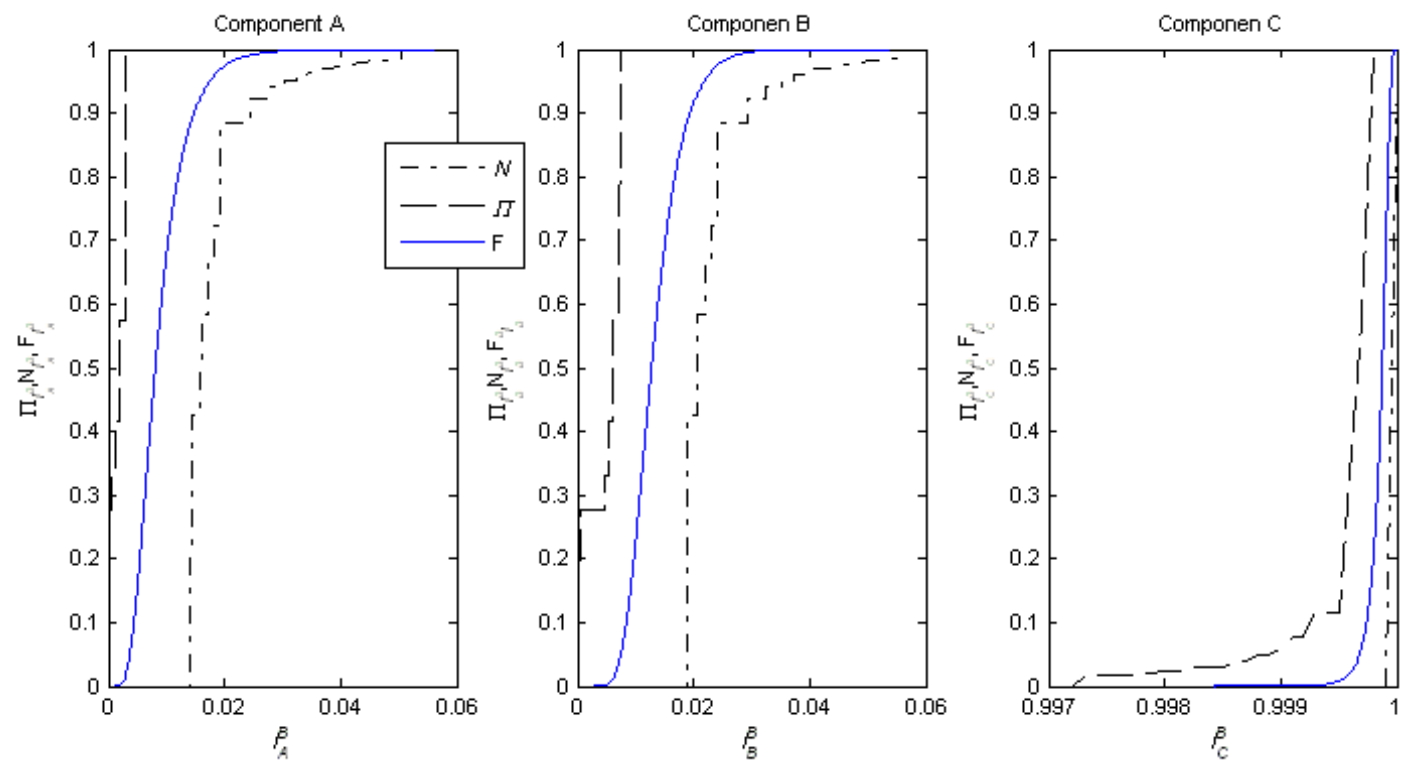

Fig. 2. Possibility measure, necessity measure, and CDF of the IMs of the components. 

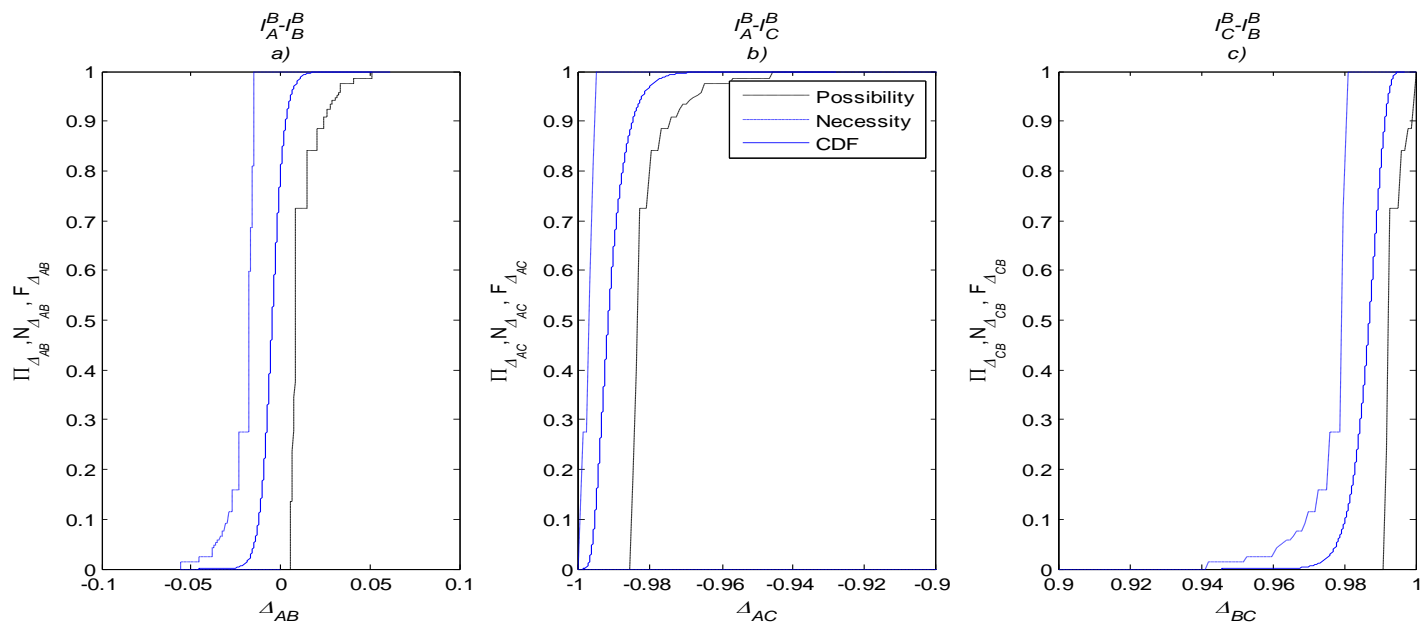

Fig. 3. Comparison of the possibility measure, necessity measure, and CDF of the variables $\Delta_{k j} k, j=A, B, C$ and $k \neq j$.

\section{Components importance ranking, under a possibilistic representation \\ of uncertainty}

Experts may not be willing to specify probability distributions of the parameters of the system model such as the component failure probabilities $\mathbf{Q}(t)=\left(Q_{1}(t), Q_{2}(t), \ldots, Q_{n}(t)\right)$ when the available information is incomplete, sparse, conflicting, vague, or non-specific [39]. Possibility theory has been proposed to deal with epistemic uncertainty in situations characterized by insufficient knowledge on parameter values.

Section 3.1 briefly introduces PT, and shows how to obtain a possibility distribution in practical cases; Section 3.2 deals with the propagation of the uncertainty from the component failure probability to the component Birnbaum importance; finally, Section 3.3 illustrates the proposed novel procedure for ranking the component Birnbaum importance.

\subsection{Possibilistic representation of uncertainty in component unreliabilities}

Let us consider the generic variable $Q$; in PT, uncertainty in this variable is represented by means of a possibility function $\pi_{Q}(q)$, which expresses the degree of possibility of each value $q$ of the variable $Q$ in a set $S$ of being the true (but unknown) value of $Q$. When $\pi_{Q}(q)=0$, it means that the outcome $q$ is considered an impossible situation. When $\pi_{Q}(q)=1$, it means that the outcome $q$ is possible, i.e., 
unsurprising, typical, usual [32]. These values are mutually exclusive becaue the uncertain variable can assume one true value only. This result also gives the normalization condition $\exists q \in S: \pi_{Q}(q)=1$, which claims that at least one value is viewed as totally possible, a much weaker statement than when the probability is $1.0[33]$.

A possibility distribution may also be viewed as a nested set of confidence intervals, which are the $\alpha$-cuts $[\underline{q}, \bar{q}]_{\alpha}=\left\{q \mid \pi_{Q}(q) \geq \alpha\right\}$ of $\pi$. The degree of certainty that $[\underline{q}, \bar{q}]_{\alpha}$ contains $Q$ is $N_{Q}\left([\underline{q}, q]_{\alpha}\right)=1-\alpha$ if $\pi_{Q}$ is continuous [25].

The possibility and necessity measures $\Pi_{Q}(A), N_{Q}(A)$ for all subsets $A \subseteq S$ are defined by the associated possibility distribution $\pi_{Q}(q)$ through the following maximization and minimization relationships, respectively:

$$
\begin{aligned}
& \Pi_{Q}(A)=\sup _{q \in A}\left\{\pi_{Q}(q)\right\} \\
& N_{Q}(A)=1-\Pi_{Q}(\text { not } A)=1-\sup _{q \notin A}\left\{\pi_{Q}(q)\right\}
\end{aligned}
$$

Let $\mathbf{P}_{Q}(\pi)$ be a family of probability distributions such that for all events $A, N_{Q}(A) \leq P_{Q}(A) \leq \Pi_{Q}(A)$. Then,

$$
N_{Q}(A)=\inf P_{Q}(A) \text { and } \Pi_{Q}(A)=\sup P_{Q}(A)
$$

where inf and sup are taken with respect to all probability measures in $\mathbf{P}_{Q}$ [30]. Hence, the necessity measure is interpreted as a lower limit for the probability, and the possibility measure is interpreted as an upper limit. Referring to subjective probabilities, the bounds reflect that the analyst is not able or willing to precisely assign his or her probability, and the bounds are the best he or she can do given the information available; in other words, he or she can only describe a subset of $\mathbf{P}_{Q}$ which contains his or her probability.

On this basis, we can define the upper $\overline{F_{Q}}(q)$ and lower $\underline{F}_{Q}(q)$ cumulative distribution functions such that $\forall q \in S, \underline{F_{Q}}(q) \leq F_{Q}(q) \leq \overline{F_{Q}}(q)$, with $\left.\left.\underline{F_{Q}}(q)=N_{Q}(]-\infty, q\right]\right)$, and $\left.\left.\overline{F_{Q}}(q)=\Pi_{Q}(]-\infty, q\right]\right)$ (i.e., the generic set $A$ 
in (4)-(6) assumes here the form of $]-\infty, q]$ ). For the sake of brevity, in the present work the possibility, and necessity measures $\left.\left.\Pi_{Q}(]-\infty, q\right]\right)$ and $\left.\left.N_{Q}(]-\infty, q\right]\right)$ are indicated with abuse of notation by $\Pi_{Q}(q)$, and $N_{Q}(q)$, respectively. For further theoretical details, the interested reader may refer to [30]-[33].

Various approaches for constructing possibility distributions have been proposed depending on the available information [25], [40], [41]. In this work, we assume that the only available knowledge on the uncertain variable $Q_{j}$ is constituted by its mean $q_{j}^{*}$, and standard deviation $\sigma_{j}$. In this case, the Chebyshev inequality can be used to build a possibility distribution [42]. Such inequality defines a bracketing approximation on the confidence intervals around the known mean $q_{j}^{*}$ of $Q_{j}$ knowing its standard deviation $\sigma_{j}$ :

$$
P\left(Q_{j} \in\left[q_{j}^{*}-a \sigma_{j}, q_{j}^{*}+a \sigma_{j}\right]\right) \geq 1-\frac{1}{a^{2}} \quad \text { for } \quad a \geq 1
$$

The Chebyshev inequality defines a possibility distribution $\pi_{Q_{j}}\left(q_{j}\right)$ by considering intervals $\left[q_{j}^{*}-a \sigma_{j}, q_{j}^{*}+a \sigma_{j}\right]$ as $\alpha$-cuts of $\pi_{Q_{j}}\left(q_{j}\right)$, and letting $\pi_{Q_{j}}\left(q_{j}^{*}-a \sigma_{j}\right)=\pi_{Q_{j}}\left(q_{j}^{*}+a \sigma_{j}\right)=1 / a^{2}$. This possibility distribution defines a probability family which has been proven to contain all distributions with mean $q_{j}^{*}$ and standard deviation $\sigma_{j}, s$-independently from the type of probability distribution, i.e, normal, lognormal, gamma, symmetric or not, unimodal or not, etc. [42].

With regard to the three-components case study of Fig. 1, we now assume that the only available knowledge is constituted by the means and standard deviations of the component failure probabilities (Table I), without any information on the type of distribution. The possibility distributions obtained by using the Chebyshev inequality are shown in Fig. 4 a, b, and c.

Fig. 4 d, e, and f show the possibility, and necessity measures $\Pi_{Q_{j}}\left(q_{j}\right)$, and $N_{Q_{j}}\left(q_{j}\right)$ obtained from the corresponding possibility distributions $\pi_{Q_{j}}\left(q_{j}\right)$ of Fig. 4 a, b, and c by using (4), and (5) respectively. In details, for a given value $q_{j}$ of $Q_{j}$, the possibility measure $\Pi_{Q_{j}}\left(q_{j}\right)$ takes the maximum value of $\pi_{Q_{j}}\left(q_{j}^{\prime}\right)$ for any $q_{j}^{\prime} \leq q_{j}$, whereas the necessity measure takes the minimum value of $1-\pi_{Q_{j}}\left(q_{j}^{\prime}\right)$ for any $q_{j}^{\prime}>q_{j}$. 
Fig. $4 \mathrm{a}, \mathrm{b}$, and c also report the $\mathrm{CDFs} F_{Q_{j}}\left(q_{j}\right)$ of the component unreliabilities considered in Section 2, for $j=A, B, C$. In this respect, notice that $F_{Q_{j}}\left(q_{j}\right) \in\left[N_{Q_{j}}\left(q_{j}\right), \Pi_{Q_{j}}\left(q_{j}\right)\right]$, for every $j=A, B, C$. This result confirms that the CDFs $F_{Q_{j}}\left(q_{j}\right)$ belong to the corresponding family $\mathbf{P}\left(\pi_{Q_{j}}\right)$ of all the probability distributions that are upper bounded by the possibility measure $\Pi_{Q_{j}}\left(q_{j}\right)$, and lower bounded by the necessity measure $N_{Q_{j}}\left(q_{j}\right)$.

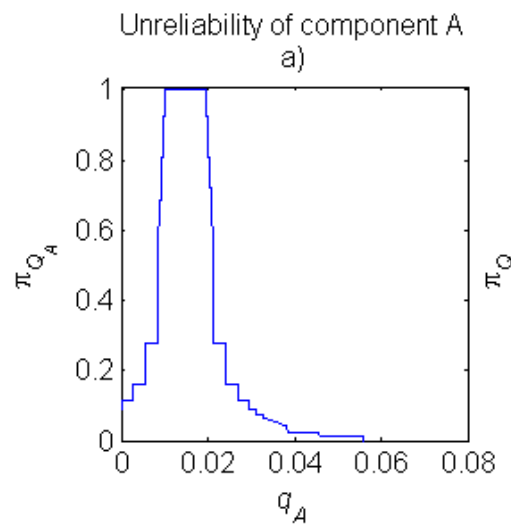

Unreliability of component B

Unreliability of component C

b)
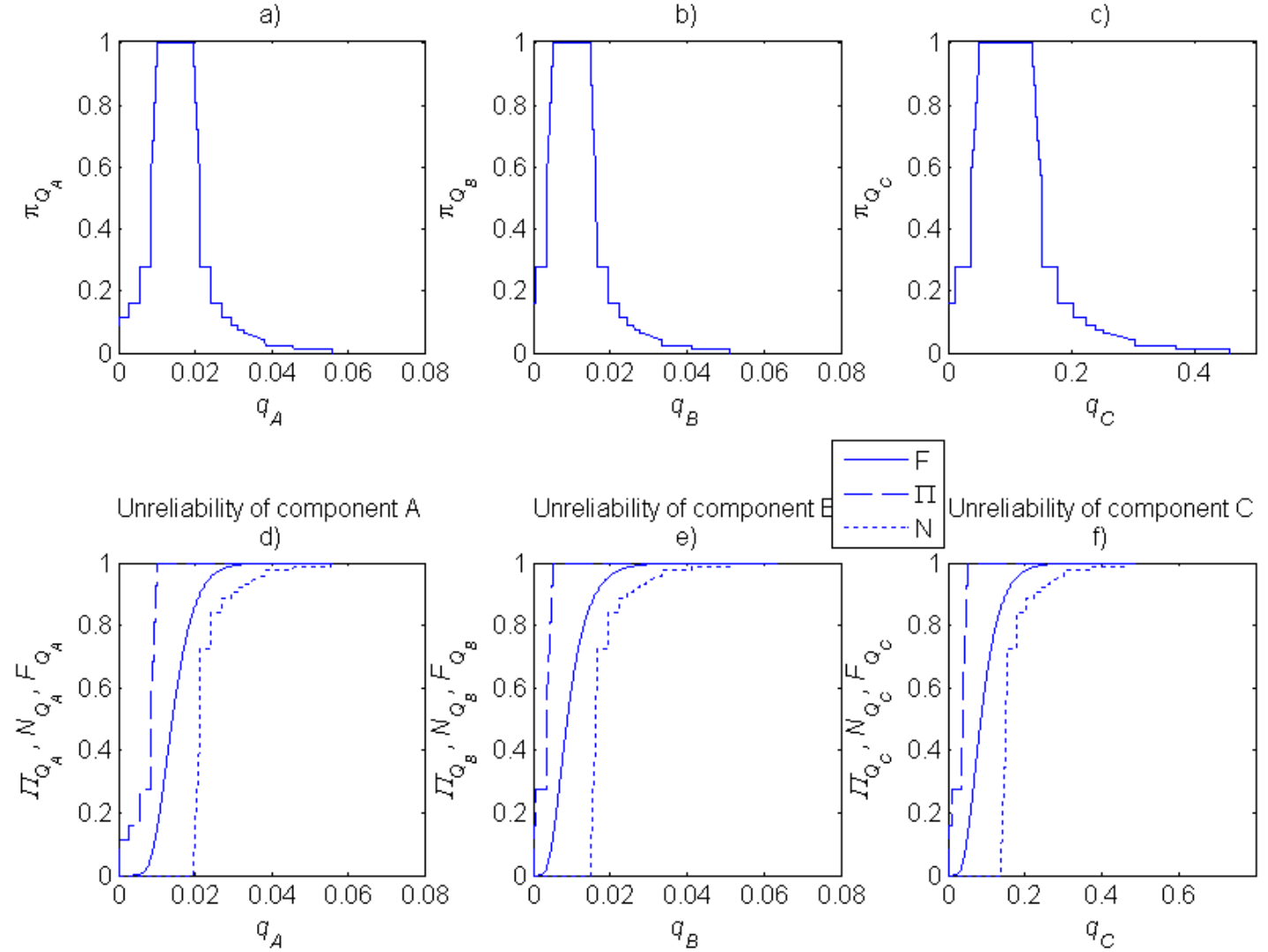

Fig. 4. a,b,c: possibility distributions of the unreliabilities of the components A,B, and C, respectively. d,e,f: possibility (dashed lines), and necessity (dotted lines) measures of the corresponding component unreliabilities. The continuous lines refers to the CDF of the component unreliability obtained in Section $\mathbf{2}$ in the probabilistic framework. The scales of the abscissas of $a$ and $b$ are different from that used in c.

\subsection{Possibilistic representation of uncertainty in component Birnbaum IMs}

To propagate the epistemic uncertainty from the component unreliabilities onto the component importance measures, the fuzzy extension principle is used [29]. In practice, the following steps are performed. 
1. Select a value of $\alpha$ on [0,1], and take as the interval of possible values of the reliabilities of the components the cut $\left[\underline{Q_{j}}, \overline{Q_{j}}\right]_{\alpha}=\left\{q_{j} \mid \pi_{Q_{j}}\left(q_{j}\right) \geq \alpha\right\}, j=A, B, C$. For example, if $\alpha=1$, then the 1 -cut of the distributions $\quad \pi_{Q_{A}}\left(q_{A}\right), \quad$ and $\quad \pi_{Q_{B}}\left(q_{B}\right) \quad$ are $\quad\left[\underline{Q_{A}}, \overline{Q_{A}}\right]_{\alpha=1}=\left[9.98 \mathrm{e}^{-3}, 19.7 \mathrm{e}^{-3}\right], \quad$ and $\left[\underline{Q_{B}}, \overline{Q_{B}}\right]_{\alpha=1}=\left[5.00 \mathrm{e}^{-3}, 14.8 \mathrm{e}^{-3}\right]$, respectively (Fig. $4 \mathrm{a}$, and b).

2. For every component of the vector $\mathbf{I}^{B}$, compute the smallest, and largest values of the function $g_{j}(\mathbf{Q})$ encoding the relationships between the failure probabilities and the Birnbaum IM of the $j$-th component (denoted by ${\underline{g_{j}}}_{\alpha}^{I_{j}^{B}}$, and ${\overline{g_{j}}}_{j_{\alpha}}^{I_{j}}$, respectively), when the elements of $\mathbf{Q}$ range within the intervals $\left[\underline{Q_{j}}, \overline{Q_{j}}\right]_{\alpha}$; that is, calculate

$$
\begin{aligned}
& \frac{\underline{g}_{j}{ }_{\alpha}^{B}}{I_{j}^{B}}=\inf _{l, q_{l} \in\left[\underline{Q_{l}}, \bar{Q}_{l}\right]_{\alpha}} g_{j}(\mathbf{Q}) \\
& \bar{g}_{j_{\alpha}}^{I_{j}^{B}}=\sup _{l, q_{l} \in\left[\underline{Q}_{l}, \bar{Q}_{l}\right]_{\alpha}} g_{j}(\mathbf{Q})
\end{aligned}
$$

These results are the lower, and upper bounds, respectively, of the $\alpha$-cut of the possibility distributions $\pi_{I_{j}^{B}}\left(i_{j}^{B}\right), j=A, B, C$.

For example, with reference to the component $C$ and (3), we have

$$
\begin{gathered}
{\underline{g_{C}}}_{\alpha=1}^{I_{C}^{B}}=\inf _{l, q_{l} \in\left[\underline{Q}_{l}, \bar{Q}_{l}\right]_{\alpha=1}} g_{C}(\mathbf{Q})=\inf _{\substack{q_{A} \in\left[9.98 \mathrm{e}^{-3}-1,7.7 \mathrm{e}^{-3}\right] \\
q_{B} \in\left[.00 \mathrm{e}^{-3}, 14.8 e^{-3}\right]}} 1-Q_{A} Q_{B}=1-19.7 * 14.8 e^{-6}=0.99970 \\
\bar{g}_{C \alpha=1}^{I_{C}^{B}}=\sup _{l, q_{l} \in\left[\underline{Q}_{l}, \bar{Q}_{l}\right]_{\alpha=1}} g_{C}(\mathbf{Q})=\sup _{\substack{q_{A} \in\left[9.9 \mathrm{e}^{-3}, 19.7 \mathrm{e}^{-3}\right] \\
q_{B} \in\left[5.00 \mathrm{e}^{-3}, 11.8 e^{-3}\right]}} 1-Q_{A} Q_{B}=1-9.98 * 5.00 e^{-6}=0.99995
\end{gathered}
$$

3. Repeat steps 1-2 for another value of $\alpha$.

4. Build the possibility, and necessity measures $\Pi_{I_{j}^{B}}\left(i_{j}^{B}\right)$, and $N_{I_{j}^{B}}\left(i_{j}^{B}\right)$ of the IMs $I_{j}^{B}$ using (4), and (5).

Fig. $3 \mathrm{a}, \mathrm{b}$, and c show the obtained possibility and necessity measures in the considered example. 


\subsection{Components Importance Ranking}

In this section, we present a procedure for ranking the possibility distributions representing the uncertainty in the Birnbaum IM of the system components. If, for example, we consider the possibility and necessity measures of components $A, B, C$, Figs. $3 \mathrm{a}, \mathrm{b}$, and c show that the upper limit (possibility measure) of the probability that the importance of component $C$ is smaller than 0.9 is 0 , whereas the lower limit of the probabilities that the importance of components $A$ and $B$ are larger than 0.06 is 0 . Thus, in this case one can conclude that component $C$ is certainly more important than components $A$ and $B$.

On the other side, ranking the IMs of components $A$ and $B$ is not straightforward, as their possibility and necessity measures overlap. This case calls for the development of a general procedure to rank the possibilistic IMs of the components. To this aim, the ranking procedure discussed in [19] in the probabilistic framework is modified to take into account that the IMs are not represented by probability distributions but by families of probability distributions whose upper, and lower bounds are the possibility, and necessity measures, respectively. The procedure proposed in this work for the ranking of the IMs is based on the following steps.

1. Compute the possibility distributions $\pi_{\Xi_{k s}}\left(\xi_{k s}\right)$ of the variable $\Xi_{k s}=\Xi_{k s}\left(I_{k}^{B}, I_{s}^{B}\right)$, defined as

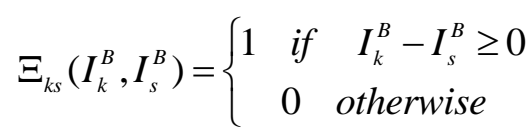

This calculation requires one to repeat the following steps for different values of $\alpha$ in the range $[0,1]$.

1.1. Select a value of $\alpha$, and identify the bounds of the $\alpha$-cut of the possibility distributions $\pi_{\Delta_{k s}}\left(\delta_{k s}\right)$, $k \neq s$, of the variables $\Delta_{k s}=I_{k}^{B}-I_{s}^{B}$ which represent the differences between the IMs of the components. These values are given by $\left[\underline{g_{k}}{ }_{\alpha}^{I_{k}^{B}}-\bar{g}_{s_{\alpha}}^{I_{s}^{B}}, \bar{g}_{k_{\alpha}}^{I_{k}^{B}}-{\underline{g_{s}}}_{I_{\alpha}}^{I_{s}^{B}}\right]$. In the example of Fig. 1, if $k=A$, and $s=C$, then $\pi_{\Delta_{A C}}\left(\delta_{A C}\right)=1$ for $\delta_{A C}$ ranging within the interval $\left[\underline{g_{A}}{ }_{\alpha=1}^{I_{A}^{B}}-\bar{g}_{C}^{I_{C=1}^{B}}, \bar{g}_{A \alpha=1}^{I_{A}^{B}}-\underline{g}_{C=1}^{I_{C}^{B}}\right]$ $=[0.00320-0.99995,0.01420-0.99970]=[-0.9950,-0.9860]$ (in Fig. 3, these values are the smallest values of $\Delta_{A C}$ in which $\Pi_{\Delta_{A C}}\left(\delta_{A C}\right)=1$, and the largest in which $N_{\Delta_{A C}}\left(\delta_{A C}\right)=0$, respectively). 
1.2. Identify the $\alpha$-cut of $\pi_{\Xi_{k s}}\left(\xi_{k s}\right)$ for every $k, s, k \neq s$; in this regard, notice that $\pi_{\Xi_{k s}}(0)=\alpha$ if $\underline{g}_{\alpha}^{I_{k}^{B}}-\bar{g}_{s \alpha}^{I_{s}^{B}}<0$, and $\pi_{\Xi_{k s}}(1)=\alpha$ if $\bar{g}_{\alpha}^{I_{k}^{B}}-\underline{g}_{\alpha}^{I_{s}^{B}} \geq 0$. In the reference example, we can see that the possibility $\pi_{\Xi_{A C}}(0)$ that $\Xi_{A C}=0$ is 1 , whereas $\pi_{\Xi_{A C}}(1)=0$.

2. Considering the lower threshold $T_{l}$ defined in Section 2.1, the relation order between the IMs of components $k$ and $s$ is established on the basis of the following criteria.

i. If $\pi_{\Xi_{k s}}(0) \leq T_{l}$, then component $k$ is more important than $s$.

ii. If $\pi_{\Xi_{k s}}(1) \leq T_{l}$, then component $s$ is more important than $k$.

iii. Components $k$ and $s$ are equally important in the other cases.

These criteria are justified by the following considerations.

- $\pi_{\Xi_{k s}}\left(\xi_{k s}\right)=1$ for at least one out of the two values of $\xi_{k s}$ (i.e., 0 and 1), being the distributions $\pi_{\Xi_{k s}}$ normalized (i.e., there must be at least one point of the Universe of Discourse (UoD) in which the distribution reaches 1). Thus, it is not possible that both the above conditions $\mathrm{i}$ and ii are contemporarily verified.

- Let us consider a case in which $\pi_{\Xi_{k s}}(0)=0.2$, and $\pi_{\Xi_{k s}}(1)=1$. Equations (4) and (5) allow stating that $\circ \quad N_{\Xi_{k s}}(0)=0$ and $\Pi_{\Xi_{k s}}(0)=0.2$, which can be probabilistically interpreted as $0 \leq P_{\Xi_{k s}}(0)=P\left(I_{k}^{B}<I_{s}^{B}\right) \leq \Pi_{\Xi_{k s}}(0)=0.2 ;$ and

$\circ \quad N_{\Xi_{k s}}(1)=0.8$, and $\Pi_{\Xi_{k s}}(1)=1$, which can be probabilistically interpreted as $0.8 \leq P_{\Xi_{k s}}(1)=P\left(I_{k}^{B} \geq I_{s}^{B}\right) \leq \Pi_{\Xi_{k s}}(1)=1$

To sum up, the probability that component $k$ is more important than component $s$ lies in the interval $[0.8,1]$, whereas the probability of the opposite case is a value between 0 and 0.2 . In this situation, in which we are confident of the relevance of $k$ with respect to $s$, it is reasonable to decide in favor of component $k$.

According to the proposed criteria, the result of the comparison of the importance of two components, $k$ and $s$, does not depend on the order in which they are considered because the possibility distributions 
$\pi_{\Xi_{k s}}(\xi)$ of the variable $\Xi_{k s}=I_{k}^{B}-I_{s}^{B}$ corresponds to the possibility distribution $\pi_{\Xi_{s k}}$ of $\Xi_{s k}=I_{s}^{B}-I_{k}^{B}$ evaluated in $-\xi$, i.e., $\pi_{\Xi_{k s}}$ and $\pi_{\Xi_{s k}}$ are symmetric with respect to the ordinate axis [44]. Thus, as it is shown in Fig. 5, if component $k$ results more important than component $s$ computing the distribution of $\Xi_{k s}=I_{k}^{B}-I_{s}^{B}$, then component $s$ always results less important than component $k$ computing the distribution of $\Xi_{s k}=I_{s}^{B}-I_{k}^{B}$

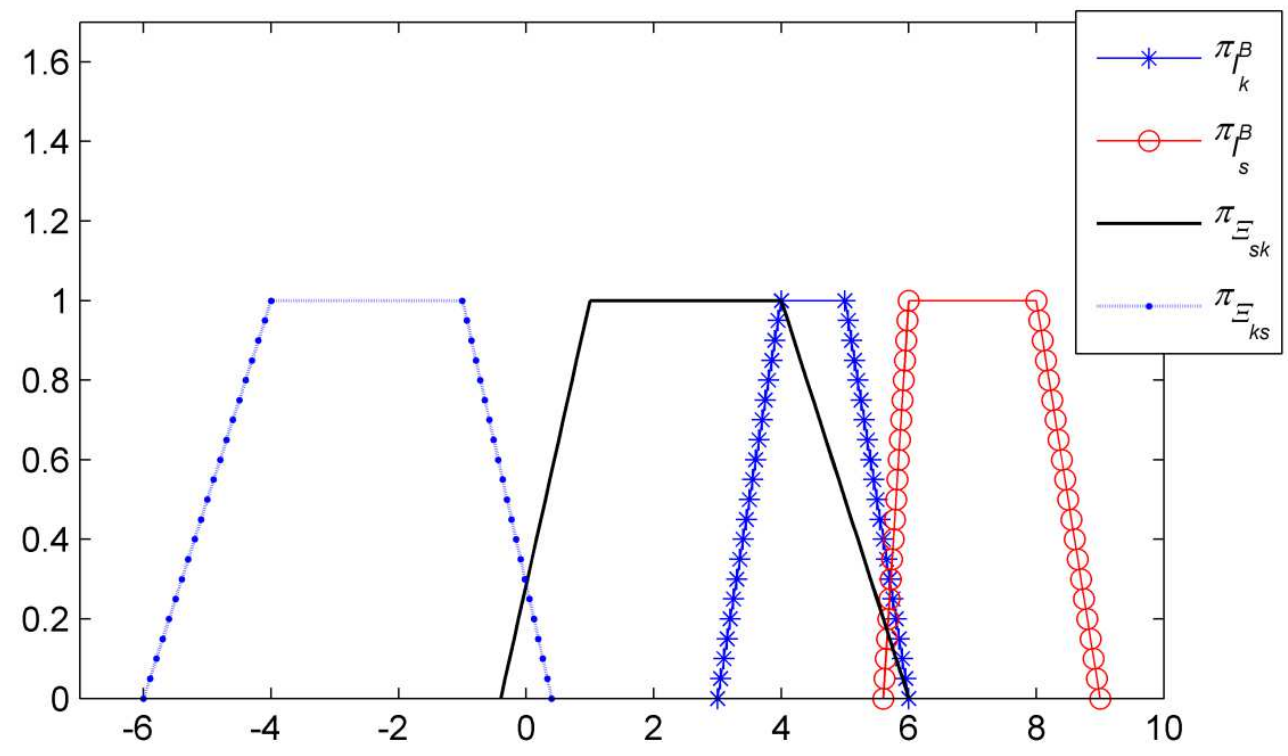

Fig. 5. Possibility distributions of the IMs of two generic components $k$ and $s$, and corresponding possibility distributions of the variables $\Xi_{s k}=I_{s}^{B}-I_{k}^{B}$ and $\Xi_{k s}=I_{k}^{B}-I_{s}^{B}$.

The results of the application of the proposed procedure to the Birnbaum IMs of the three components $A, B$, and $C$ of Fig. 1 are reported in Fig. 6. Fig. 6. Component $C$ turns out to be the most important, whereas components $A$ and $B$ are equally important. This final ranking is different from that obtained in the probabilistic framework (Section 2.1), where component $B$ is judged more important than component $A$. This result is due to the fact that in the probabilistic framework the analyst focuses on one out of the infinite probability distributions encompassed by a possibility distribution. Thus, as expected, the final ranking derived from the uncertain IMs depends on the information available: if the analyst believes that resorting to the probability theory framework is justified, then he or she will be capable of assessing that component $B$ is more important than component $A$; whereas, in the opposite case, if the analyst is not able to specify a single 
probability distribution but prefers to use a possibility distribution, then he or she has not enough information to conclude that one component is more important than the other.

Analogously to the probabilistic case, if we consider the problem of ranking the importance of three generic components, $j, k, s$, one may obtain a contradictory ranking which does not obey the transitive property 'if $I_{j}^{B}>I_{k}^{B}$ and $I_{k}^{B}>I_{s}^{B}$, then $I_{j}^{B}>I_{s}^{B}$,. However, as in the probabilistic framework, setting $T_{l}$ smaller than $1 / 3$ allows avoiding the contradictory ranking with $I_{j}^{B}>I_{k}^{B}, I_{k}^{B}>I_{s}^{B}$, and $I_{s}^{B}>I_{j}^{B}$, but can lead to undesirable situations in which $I_{j}^{B}>I_{k}^{B}, I_{k}^{B}>I_{s}^{B}$, and $I_{j}^{B}=I_{s}^{B}$, or similarly, $I_{j}^{B}>I_{k}^{B}, I_{k}^{B}=I_{s}^{B}$, and $I_{j}^{B}=I_{s}^{B}$ (see the Appendix for the mathematical details). These situations are addressed by assuming that the three components have the same ranking. i.e., $I_{j}^{B}=I_{s}^{B}=I_{k}^{B}$.

In the case in which the system is made by several components, a sorting algorithm needs to be used to automatically order the components according to their importance. To this aim, a number of algorithms can be found in the literature, which have different computational complexities, memory usage strategies, etc. [23], [45]. They usually sort the component IMs by performing a limited number of all the possible pairwise comparisons. However, whichever is the sorting algorithm chosen to arrange the components' IMs in ascending order, it needs to be modified to address the case, previously outlined, in which $I_{j}^{B}>I_{k}^{B}, I_{k}^{B}>I_{s}^{B}$, and $I_{j}^{B}=I_{s}^{B}$, which may lead to different rankings depending on which pairwise comparisons are performed. For example, if the ranking algorithm compares $j$ with $k$, and $k$ with $s$, but it does not compare $j$ with $s$, it will produce a ranking with $I_{j}^{B}>I_{k}^{B}>I_{s}^{B}$; whereas, if it compares $j$ with $k$, and $j$ with $s$, but not $k$ with $s$, it will rank $I_{j}^{B}=I_{s}^{B}>I_{k}^{B}$. To avoid that the obtained final rank depends from which comparisons are made by the ranking algorithm, it is necessary to apply to the components ranking proposed by the sorting algorithm an additional control procedure which checks whether equally important components occupy different positions in the ranking. In this situation, the final ranking is correspondingly modified by assigning the same rank to all the involved components. The Appendix describes one of the most used sorting algorithm, i.e., the Quicksort algorithm [23], and proposes a control procedure to verify whether equally important components occupy different positions in the ranking. 

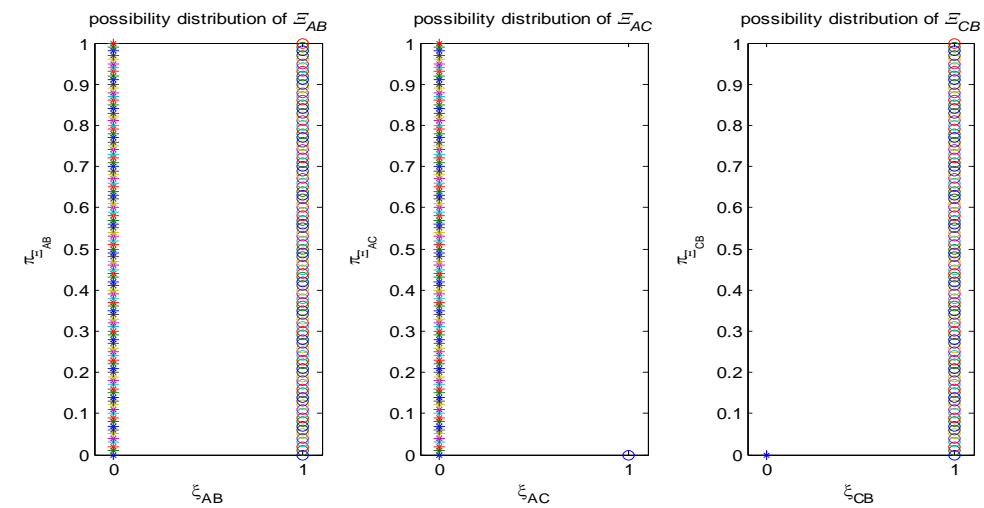

Fig. 6. Comparison of the Importance measures: possibility distributions of the variables $\Xi_{k j} k, j=A, B, C$, and $k \neq j$.

\section{Industrial case study: Auxiliary Feedwater System}

Let us consider an AFWS of a Pressurized Water Reactor (PWR) whose simplified Reliability Block Diagram (RBD) is reported in Fig. 7. The case study is taken from [18], where it is assumed that

a) all components are in standby mode,

b) all components are periodically tested, and

c) the components' unavailabilities $Q_{j}$ are affected by epistemic uncertainties which are described by lognormal probability distributions.

In this work, instead of assumption c, we consider a case in which the only available knowledge on the $Q_{j}$ values is constituted by their mean and standard deviation values (Table ) without any information on the type of probability distributions. To allow a comparison with a probabilistic approach, the values in Table II correspond to the mean and standard deviations of the lognormal distributions used in [18] to represent the uncertainty in the $Q_{j}$ values within a probabilistic approach.

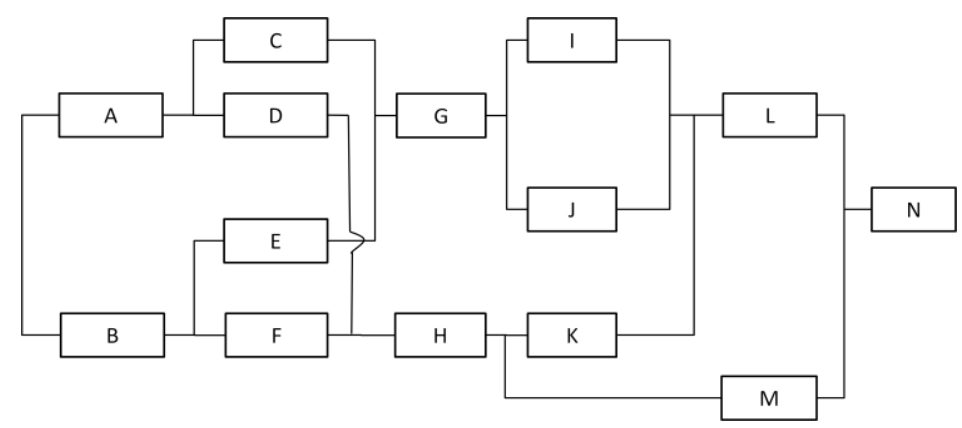

Fig. 7. RBD of the AFWS system [18]. 


\begin{tabular}{|c|c|c|}
\hline $\begin{array}{c}\text { Component } \\
\text { Name }\end{array}$ & Mean unavailability $q_{j}^{*}$ & Standard deviation $\sigma_{j}$ \\
\hline$A$ & $9,963 \mathrm{E}-05$ & $1,787 \mathrm{E}-05$ \\
\hline$B$ & $9,963 \mathrm{E}-05$ & $1,7875 \mathrm{E}-05$ \\
\hline$C$ & $70.22 \mathrm{E}-05$ & $17.750 \mathrm{E}-05$ \\
\hline$D$ & $70.22 \mathrm{E}-05$ & $17.750 \mathrm{E}-05$ \\
\hline$E$ & $70.22 \mathrm{E}-05$ & $17.750 \mathrm{E}-05$ \\
\hline$F$ & $70.22 \mathrm{E}-05$ & $17.750 \mathrm{E}-05$ \\
\hline$G$ & $5,129 \mathrm{E}-05$ & $1,762 \mathrm{E}-05$ \\
\hline$H$ & $4,080 \mathrm{E}-05$ & $1,740 \mathrm{E}-05$ \\
\hline$I$ & 0,07088 & 0,01705 \\
\hline$J$ & 0,07088 & 0,01705 \\
\hline$K$ & 0,02458 & 0,001735 \\
\hline$L$ & $23.72 \mathrm{E}-05$ & $8,875 \mathrm{e}-05$ \\
\hline$M$ & 0,10858 & 0,053250 \\
\hline$N$ & $3,9778 \mathrm{E}-05$ & $1,7875 \mathrm{E}-05$ \\
\hline
\end{tabular}

The uncertainties in the components' unavailabilities $Q_{A}, \ldots, Q_{N}$ are propagated by the procedure illustrated in Section 3.2, and the possibility and necessity measures of Birnbaum IMs of the components are obtained (Fig. 8). As expected, the largest Birnbaum IM is assigned to component $N$, which is a single point failure (i.e., its failure results in the loss of AWFS functionality), and thus the overall AWFS reliability is strongly sensitive to the improvement of the reliability of this component.

Because the AFWS has a large number of components, the Quicksort algorithm has been used to automatically order the components on the basis of their importance. Tables III and IV report two different rankings obtained by applying the Quicksort algorithm with two different initial settings. According to Table III, there is a first group of elements $(F C D E)$ whose IMs are sensibly smaller than those of the components of the second group $(A B I J M)$. Another group $(G H K)$ of components with similar importance values has been identified; these are less important than $L$ (the second most important component), which is sensibly less important than $N$. Differently, in the case of the Quicksort execution reported in Table IV, the direct comparison between the Birnbaum IMs of components $K$ and $I$ is performed, and the two components turn 
out to be equally important; this result leads to assigning the same importance to the components $A B I J M G$ $H K$.

Table III

Ranking of the components' IMs obtained in the first execution of the Quicksort algorithm, and confirmed after applying the procedure to verify whether equally important components occupy different positions (possibilistic framework)

\begin{tabular}{lcccccc}
\hline Ranking order & 14-13-12-11 & 10-9-8-7-6 & $5-4-3$ & 2 & 1 \\
\hline Components & FDCE & A B IJ M & GHK & $L$ & $N$ \\
& & & & & \\
\hline
\end{tabular}

Table IV

Ranking of the components' IMs obtained in the second execution of the Quicksort algorithm (possibility theory framework)

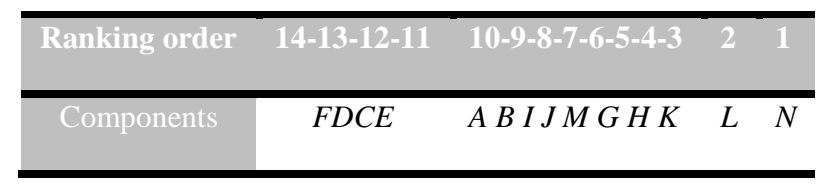

To avoid such instability of the importance ranking, according to the procedure described in the Appendix, all the possible comparisons between the components of the system under study are performed (TableV). In the case of Table III, the components' ranking is modified to take into account that $I_{K}^{B}=I_{I}^{B}$, and the final ranking becomes that of Table IV, whereas in the second run of the Quicksort algorithm (Table IV), the proposed ranking does not need to be modified. 

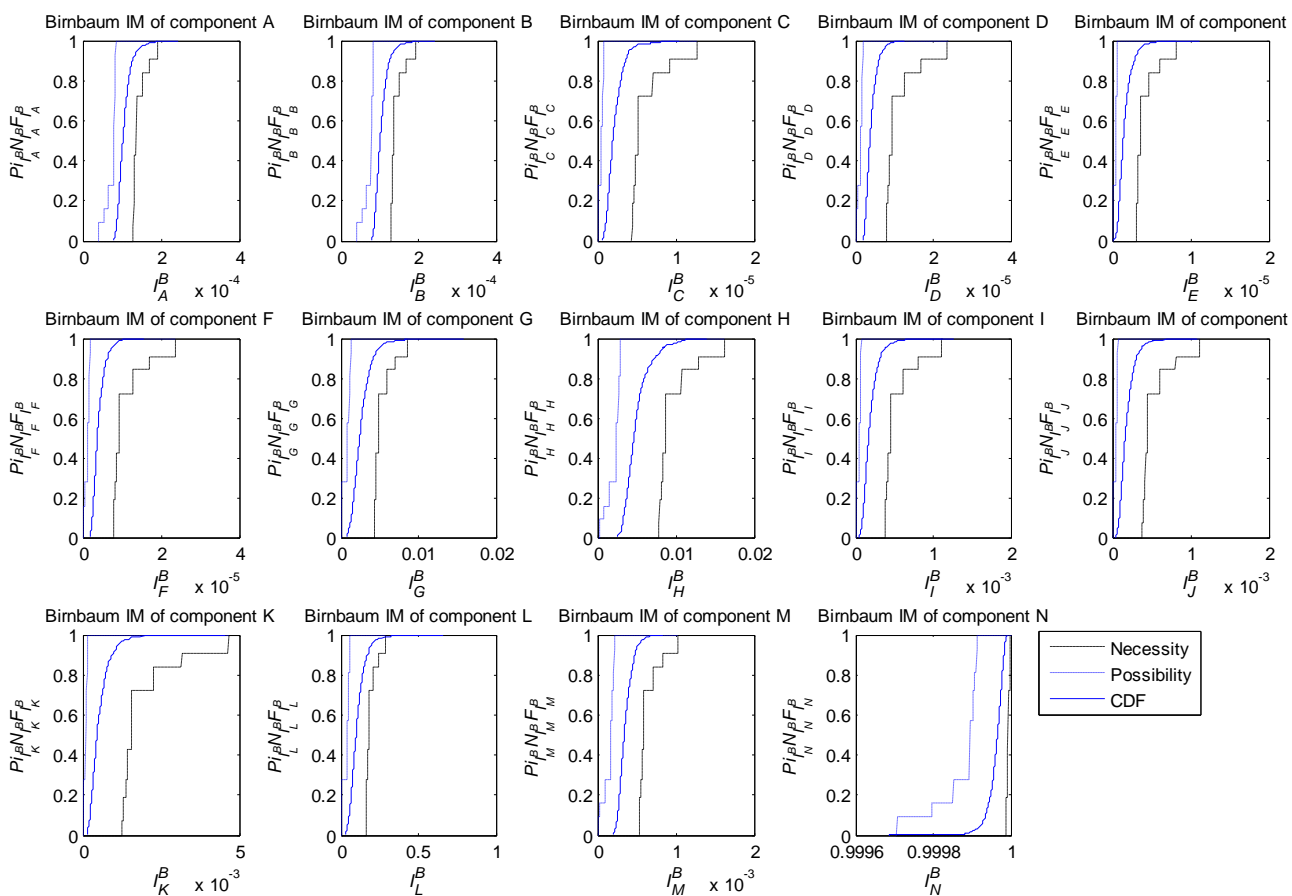

Fig. 8. Possibility, and necessity measures of the components' Birnbaum IMs (reported in different scales.). The continuous line

refers to the CDFs obtained in the probabilistic case [14].

TableV

Results of the comparisons between the Birnbaum IMs of the components of the AFWS, according to the ranking criterion discussed in Section 3.3. The symbols in the matrix describe the relation order between the elements in the rows and those in the columns. For example, the < symbol in the first row, fifth column states that component $F$ is less important than component B.

\begin{tabular}{|c|c|c|c|c|c|c|c|c|c|c|c|c|c|c|}
\hline & $\mathrm{F}$ & D & $\mathrm{C}$ & $\mathrm{E}$ & B & A & $\mathrm{J}$ & $\mathrm{M}$ & I & $\mathrm{G}$ & $\mathrm{K}$ & $\mathrm{H}$ & $\mathrm{L}$ & $\mathrm{N}$ \\
\hline $\mathrm{F}$ & & $=$ & $=$ & $=$ & $<$ & $<$ & $<$ & $<$ & $<$ & $<$ & $<$ & $<$ & $<$ & $<$ \\
\hline $\mathrm{D}$ & & & $=$ & $=$ & $<$ & $<$ & $<$ & $<$ & $<$ & $<$ & $<$ & $<$ & $<$ & $<$ \\
\hline C & & & & $=$ & $<$ & $<$ & $<$ & $<$ & $<$ & $<$ & $<$ & $<$ & $<$ & $<$ \\
\hline $\mathrm{E}$ & & & & & $<$ & $<$ & $<$ & $<$ & $<$ & $<$ & $<$ & $<$ & $<$ & $<$ \\
\hline B & & & & & & $=$ & $=$ & $<$ & $=$ & $<$ & $=$ & $<$ & $<$ & $<$ \\
\hline A & & & & & & & $=$ & $<$ & $=$ & $<$ & $=$ & $<$ & $<$ & $<$ \\
\hline $\mathrm{J}$ & & & & & & & & $=$ & $=$ & $<$ & $=$ & $<$ & $<$ & $<$ \\
\hline M & & & & & & & & & $=$ & $>$ & $=$ & $>$ & $<$ & $<$ \\
\hline I & & & & & & & & & & $<$ & $=$ & $<$ & $<$ & $<$ \\
\hline $\mathrm{G}$ & & & & & & & & & & & $=$ & $=$ & $<$ & $<$ \\
\hline $\mathrm{K}$ & & & & & & & & & & & & $<$ & $<$ & $<$ \\
\hline $\mathrm{H}$ & & & & & & & & & & & & & $<$ & $<$ \\
\hline $\mathrm{L}$ & & & & & & & & & & & & & & $<$ \\
\hline $\mathrm{N}$ & & & & & & & & & & & & & & \\
\hline
\end{tabular}

Table reports the results obtained in [19] applying the probabilistic approach to the same case study. The main difference is that the probabilistic approach results in fewer cases of components with the same importance because the CDFs of the components' important measures appear to be more clearly separated than are the necessity and possibility measures in the possibilistic approach. 


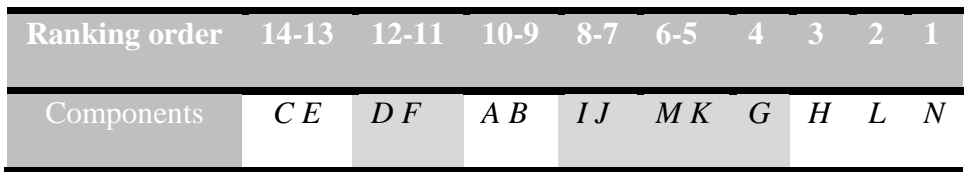

With regards to the computational times, when the epistemic uncertainties are described by means of possibility distributions, then the application of the propagation technique, the Quicksort algorithm, and the procedure to verify whether equally important components occupy different positions in the ranking take few seconds. On the contrary, the time required to carry out the same analysis within the probability theory framework is around 20 minutes, with most of the time dedicated to the uncertainty propagation step. This result is due to the fact that uncertainty propagation is performed in the possibilistic case by using the fuzzy extension principle which requires only to apply simple rules of interval algebra, whereas in the probabilistic case it is necessary to resort to MC simulation because analytical approaches are impracticable.

\section{Conclusions}

In this work, a procedure has been proposed for ranking system components in order of importance when their failure probabilities are poorly known, and the related uncertainties are described by possibility distributions. The ranking procedure is based on: i) the use of the fuzzy extension principle to propagate epistemic uncertainty from the system parameters to the component importance measures, ii) pairwise comparisons of the obtained component importance measures, and iii) the application of the Quicksort algorithm. Because the method used to compare the Birnbaum importance of three generic components, $k, s$, $j$ may lead to a ranking which can be partially contradictory $\left(I_{j}^{B} \geq I_{k}^{B}\right.$ and $I_{k}^{B} \geq I_{s}^{B}$, but $\left.I_{j}^{B} \leq I_{s}^{B}\right)$ a procedure to verify whether equally important components occupy different positions in the ranking has been proposed.

The application of the proposed procedure has shown several results. 
- We find the relevance of taking into account uncertainties in the computation of the IMs, and the fact that the possibility distributions allow to represent the uncertainties in a way less committed than that offered by the probability theory.

- The final ranking may depend on the investigation framework used to carry out the analysis, which is mainly established on the basis of the quality and quantity of available data. In general, the probabilistic representation and propagation of the uncertainty allows one to get a more refined final ranking, but it calls for a larger amount of available data and more accurate information (which may be lacking in real industrial applications).

The proposed procedure has been applied in this work to rank the components on the basis of their Birnbaum IMs, but it can be easily extended to other IMs (e.g., the Risk Reduction Worth, RRW, Risk Achievement Worth, RAW, Fussel-Vesely, FV). Notice, however, that the propagation of the epistemic uncertainty from the system parameters to the components' importance measure by using the fuzzy extension principle can become computationally more challenging when the importance measure is defined through relationships more complex than those of Birnbaum IM, and which require one to perform divisions between uncertain quantities. Furthermore, the larger the complexity of the system and the associated number of basic events, the larger is expected to be the computational time required to perform the ranking.

\section{Acknowledgment}

The authors wish to thank the anonymous reviewers for their comments and suggestions, which have been fundamental for the improvement of the paper.

\section{Appendix}

\section{Contradictory ranking (probabilistic framework)}

Let us consider a case in which we have to rank the importance of three components: $A, B$, and $C$. We have $P\left(I_{A}^{B}>I_{B}^{B}\right)>1-T_{l}$, and $P\left(I_{B}^{B}>I_{C}^{B}\right)>1-T_{l}$. According to the decision criterion of Section 2.1, these 
inequalities entail $I_{A}^{B}>I_{B}^{B}$, and $I_{B}^{B}>I_{C}^{B}$. To avoid the contradictory inequality $I_{C}^{B}>I_{A}^{B}$, we have to prove that $P\left(I_{C}^{B}>I_{A}^{B}\right)<1-T_{l}$.

To this aim, notice that $P\left(I_{C}^{B}>I_{A}^{B}\right)<1-T_{l} \Leftrightarrow 1-P\left(I_{A}^{B}>I_{C}^{B}\right)<1-T_{l} \Leftrightarrow P\left(I_{A}^{B}>I_{C}^{B}\right)>T_{l}$. Then, to prove that the contradictory inequality is not possible, we have to show that $P\left(I_{A}^{B}>I_{C}^{B}\right)>T_{l}$. Notice also that

$$
P\left(I_{C}^{B}<I_{A}^{B}\right) \geq P\left(I_{C}^{B}<I_{B}^{B}, I_{B}^{B}<I_{A}^{B}\right)
$$

because the event on the right-hand side of the equation only introduces an additional constraint on $I_{B}^{B}$, which is not considered in the left-hand side.

According to de Morgan's laws,

$$
P\left(I_{C}^{B}<I_{B}^{B}, I_{B}^{B}<I_{A}^{B}\right)=1-P\left(I_{C}^{B}>I_{B}^{B}\right)-P\left(I_{B}^{B}>I_{A}^{B}\right)+P\left(I_{C}^{B}>I_{B}^{B}>I_{A}^{B}\right)
$$

Notice that because both $P\left(I_{C}^{B}>I_{B}^{B}\right)$ and $P\left(I_{B}^{B}>I_{A}^{B}\right)$ are smaller than $T_{l}$ (by hypothesis), we obtain that

$$
P\left(I_{C}^{B}<I_{A}^{B}\right)>1-2 T_{l}+P\left(I_{C}^{B}>I_{B}^{B}>I_{A}^{B}\right)
$$

To verify that the condition $1-2 T_{l}+P\left(I_{C}^{B}>I_{B}^{B}>I_{A}^{B}\right) \geq T_{l}$, which avoids the contradictory sequence $I_{A}^{B}>I_{B}^{B}$, $I_{B}^{B}>I_{C}^{B}$, and $I_{C}^{B}>I_{A}^{B}$ is verified, it is required that $T_{l} \leq 1 / 3$, whatever is the value of $P\left(I_{C}^{B}>I_{B}^{B}>I_{A}^{B}\right)$ (in $[0,1])$.

\section{Contradictory ranking (possibilistic framework)}

Suppose that $\pi_{\Xi_{s k}}(0) \leq T_{l}$, and $\pi_{\Xi_{k j}}(0) \leq T_{l}$, which entail $\Pi_{\Xi_{s k}}(0) \leq T_{l}$, and $\Pi_{\Xi_{k j}}(0) \leq T_{l}$ (from (4) and (5) respectively). On this basis, according to the criterion propounded in Section 3.3 we can state that $I_{s}^{B}$ is larger than $I_{k}^{B}$, and $I_{k}^{B}$ is larger than $I_{j}^{B}$. To avoid the inequality $I_{j}^{B}>I_{s}^{B}$, in this case we have to show that $\pi_{\Xi_{j s}}(0)>T_{l}$.

Considering the probabilistic interpretation of the possibility distributions (6), from our hypotheses $\pi_{\Xi_{s k}}(0) \leq T_{l}$, and $\pi_{\Xi_{k j}}(0) \leq T_{l}$, we can derive 


$$
\begin{aligned}
& 0 \leq P\left(I_{s}^{B}<I_{k}^{B}\right)=P_{\Xi_{s k}}(0) \leq \Pi_{\Xi_{s k}}(0) \leq T_{l} \\
& 0 \leq P\left(I_{k}^{B}<I_{j}^{B}\right)=P_{\Xi_{k j}}(0) \leq \prod_{\Xi_{k j}}(0) \leq T_{l}
\end{aligned}
$$

Then, given the results showed earlier in this Appendix, we can guarantee that $P\left(I_{s}^{B}>I_{j}^{B}\right)>T_{l}$, if we consider a value of $\left.T_{l}<1 / 3\right)$. This result entails that $T_{l}<P\left(I_{j}^{B}<I_{s}^{B}\right) \leq \Pi_{\Xi_{j s}}(0)=\pi_{\Xi_{j s}}(0)$, and thus that $\pi_{\Xi_{j s}}(0)>T_{l}$.

\section{Ranking algorithm}

Quicksort is a divide-and-conquer algorithm which relies on a partition of the elements based on a quantitative indicator of their 'size'. To partition the elements, it is required to choose one of them as a pivot, a reference for moving all elements of size smaller before the pivot, and all elements of size larger after it. In the resulting iterative partition procedure, the sublists of smaller and larger elements are recursively sorted. The pseudo-code of the algorithm can be found in [19], and [23].

Fig 9 illustrates the application of the Quicksort algorithm in the case study of Section 5, when the components are initially arranged in alphabetical order, and the pivot is chosen as the central element of each sublist. At the first iteration, the pivot is $H$, and two sublists are created: one containing the components that are equally or more important than the pivot (right branch, in this case it contains $L$ and $N$ ), and the other with the less or equally important components (left branch, in this case it includes $A B C D E F G I J K)$. Thus, $H$ takes the third place instead of $L$, which currently occupies the second position being more important than $H$. Notice that the pairwise comparisons also show that $H$ is equally important than $G$, although the algorithm leaves $G$ in its current position. The sublist of more important components is then sorted. The comparison between $N$ and $L$ shows that the former is more important than the latter.

The less important elements branch contains $A B C D E F G M I J K$; its middle element, $F$, is chosen as pivot. The components $A B C D E F I J M$ are more or equally important than $F$. In particular, this latter component is equally important as $E, C$, and $D$. With reference to the right sublist (more important components), $G$ is the pivot element, and it swaps its position with $J$; that is, the importance of $J$ is smaller than that of $G$. The algorithm proceeds as illustrated in Fig. 9. 
Once the Quicksort algorithm has been executed, we have to run the control algorithm to verify whether different rank orders have been assigned to equally important components. This need is due to the fact that the algorithm does not perform all the possible direct comparisons.

In details, we consider the $l$-length vector $X$ of sorted components (i.e., the output of the Quicksort algorithm), and the vector $R$ of the ranking position initially associated to the components (i.e., from 1 to $l$ ). In the case considered in this appendix $X=F D C E B A J M I G K H L N$.

The rationale of the code is that to have a difference in components importance ranking there must be a column of the comparison matrix whose entries are all ' $<$ '.

We start from setting the ranking order $r=1$, and our first objective is to find the set of the least important components (i.e., all those components with rank $r=1$ ). Notice that the first component of the list provided by the sorting algorithm which does not belong to this class is the first component of the list characterized by a column of the comparison matrix Comp (TableV) containing only ' $<$, , because this is the only condition guaranteeing that all the previous components of the Quicksort list are less important. To identify this component, we set $i=2$, and check whether the $i$-th column of the comparison matrix Comp (TableV) contains only ' $<$ '. In the affirmative case, the rank of the components of $X$ from position 1 to $i-1$ is set to 1 , and the rank of component $X(i)$ is set to $i$. On the contrary, if column $i$ contains '>' or '=', then $X(i)$ and $X(1)$ must be considered of the same importance, and thus of rank 1 , because in this case $X(i)$ is equally important to at least one of the previous components. The variable $i$ is updated to $i+1$, and the procedure is iteratively repeated by considering the new column $i$ of the comparison matrix Comp.

The pseudo-code of the proposed algorithm follows.

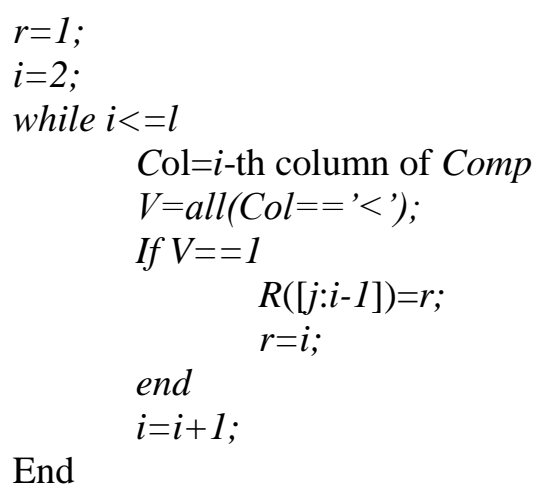

For example, consider $i=1$; then $\mathrm{X}(1)=F$. Flowing over $X$, we find that the Boolean variable $V=1$ when $i=5$. Then the first group is made up of the components $r: i-1=1: 4$ of $X$ (that is $F D C E$ ), which are of rank $r=1$.

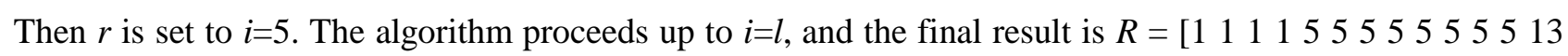
14]. 


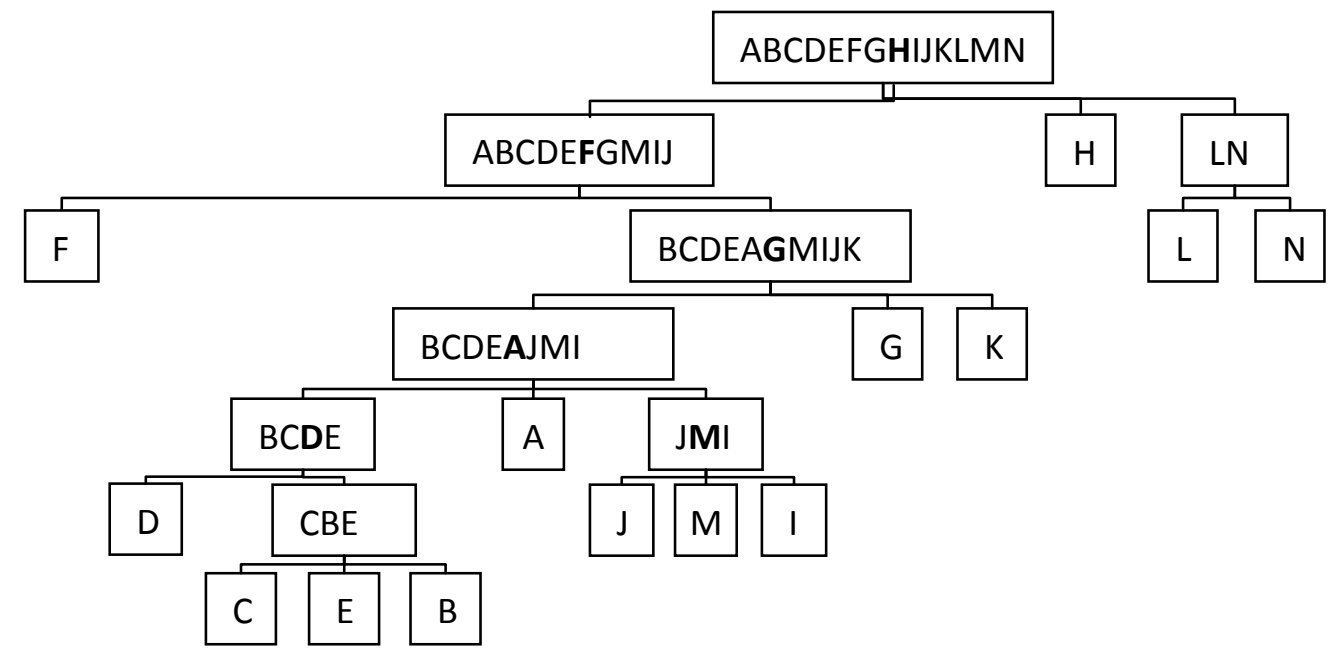

Fig. 9. steps of the Quicksort algorithm.

\section{References}

[1] T. Aven, O. Renn, and E.A. Rosa, "On the ontological status of the concept of risk," Safety Science, vol. 49, no. 8-9, pp. 1074-1079, 2011.

[2] E. Zio, Computational Methods for Reliability and Risk Analysis, Singapore: World Scientific Publishing, 2009.

[3] W. Kuo, and X. Zhu, "Some Recent Advances on Importance Measures in Reliability," IEEE Transactions on Reliability, vol. 61, no. 2, pp. 344-360, 2012.

[4] Q. Yao, X. Zhu, and W. Kuo, "A Birnbaum-importance based genetic local search for component assignment problems," Annuals of Operations Research, DOI: 10.1007/s10479-012-1223-1, 2012.

[5] X. Zhu and W. Kuo, "Importance measures in reliability and mathematical programming," Annals of Operations Research, DOI:10.1007/s10479-012-1127-0, 2012.

[6] W. Kuo and X. Zhu, "Relations and generalizations of importance measures in reliability," IEEE Transactions on Reliability, vol. 61, no. 3, pp. 659-674, 2012.

[7] W. Kuo and X. Zhu, Importance Measures in Reliability, Risk, and Optimization: Principles and Applications, Chichester, UK: John Wiley \& Sons, 2012.

[8] Z.W. Birnbaum, On the importance of different components in a multi component system. Multivariate analysis II, New York: Academic Press, 1969.

[9] R.W. Youngblood, "Risk significance and safety significance," Reliability Engineering and System Safety, vol. 73, no. 2, pp. 121-136, 2001. 
[10] S. Si, H. Dui, Z. Cai, and S. Sun, “The Integrated Importance Measure of Multi-State Coherent Systems for Maintenance Processes," IEEE Transactions on Reliability, vol. 61, no. 2, pp. 266-273, 2012.

[11] W.E. Vesely, M. Belhadj, and J.T. Rezos, "PRA importance measures for maintenance prioritization applications," Reliability Engineering and System Safety, vol. 43, no. 3, pp. 307-318, 1994.

[12] S. Martorell, V. Serradell, and G. Verdù, "Safety-related equipment prioritization for reliability centered maintenance purposes based on a plant specific level 1 PSA,” Reliability Engineering and System Safety, vol. 52, no. 1, pp. 35-44, 1996.

[13] M.C. Cheok, G.W. Parry, and R.R. Sherry, "Use of importance measures in risk-informed regulatory applications," Reliability Engineering \& System Safety, vol. 60, no. 3, pp. 213-226, 1998.

[14] H. Peng, D.W. Coit, and Q. Feng, "Component Reliability Criticality or Importance Measures for Systems With Degrading Components," IEEE Transactions on Reliability, vol. 61, no. 1, pp. 4-12, 2012

[15] S. Beeson and J.D. Andrews, "Importance Measures for Non-Coherent-System Analysis," IEEE Transactions on Reliability, vol. 42, no. 3, pp. 301-310, 2003

[16] Q. Yao, X. Zhu, and W. Kuo, "Heuristics for component assignment problems based on the Birnbaum importance," IIE Transactions, vol. 43, no. 9, pp. 1-14, 2011.

[17] G.E. Apostolakis, "The concept of probability in safety assessments of technological systems," Science, vol. 250, no. 4986, pp. 1359-1364, 1990.

[18] M. Modarres, Risk Analysis in Engineering: Probabilistic Techniques, Tools and Trends, CRC Press, 2006.

[19] P. Baraldi, E. Zio, and M. Compare, "A method for ranking components importance in presence of epistemic uncertainties," Journal of Loss Prevention in the Process Industries, vol. 22, no. 5, pp. 582-592, 2009.

[20] T. Aven and T.E. Nøkland, "On the use of uncertainty importance measures in reliability and risk analysis," Reliability Engineering System Safety, vol. 95, no. 2, pp. 127-133, 2010.

[21] E. Borgonovo, "Measuring Uncertainty Importance: investigation and comparison of alternative approaches," Risk Analysis, vol. 26, no. 5, pp. 1349-61, 2006. 
[22] E. Borgonovo, G.E. Apostolakis, S. Tarantola, and A. Saltelli, "Comparison of global sensitivity analysis techniques and importance measures in PSA," Reliability Engineering \& System Safety, vol. 79, no. 2, pp. 175-186, 2003.

[23] C.A Hoare, “Quicksort,” Computer Journal, vol. 5, no. 1, pp. 10-15, 1962.

[24] D. Dubois, H. Prade, and P. Smets, "Representing partial ignorance," IEEE Transaction System Man, Cybernetic, vol. 26, no. 3, pp. 361-377, 1996.

[25] C. Baudrit, D. Dubois, and D. Guyonnet, "Joint Propagation and Exploitation of Probabilistic and Possibilistic Information in Risk Assessment," IEEE transactions on fuzzy systems, vol. 14, no. 5, pp. 593$608,2006$.

[26] D. Dubois, L. Foulloy, G. Mauris, and H. Prade, "Probability-Possibility Transformation, Triangular Fuzzy Sets, and Probabilistic Inequalities,” Reliable Computing, vol. 10, no. 4, pp. 273-297, 2004.

[27] J.C. Helton, J.D. Johnson, and W.L., Oberkampf, "An exploration of alternative approaches to the representation of uncertainty in model predictions," Reliability Engineering and System Safety, vol. 85, no. 1-3, pp. 39-71, 2004.

[28] G. Shafer, A mathematical theory of evidence, Princeton University Press, Princeton, NJ, 1976.

[29] L.A Zadeh, “Fuzzy Sets,” Information and Control, vol. 8, no. 3, pp. 338-353, 1965.

[30] C. Baudrit, D. Dubois, and N. Perrot, "Representing parametric models tainted with imprecision," Fuzzy Sets and Systems, vol. 159, no. 15, pp. 1913-1928, 2008.

[31] C. Baudrit and D. Dubois, "Comparing methods for joint objective and subjective uncertainty propagation with an example in a risk assessment," in 4th International Symposium on Imprecise Probabilities and Their Applications, Pittsburgh, Pennsylvania, 2005.

[32] D. Dubois, "Possibility Theory and Statistical Reasoning," Computational Statistics and Data analysis, vol. 51, no. 1, pp. 47-69, 2006.

[33] D. Dubois and H. Prade, "Possibility theory, probability theory and multiple valued-logics: A clarification,” Annals o Mathematics in Artificial Intelligence, vol. 32, no. 1-4, pp. 35-66, 2001.

[34] D. Dubois and H. Prade, Possibility Theory: An Approach to Computerized Processing of Uncertainty, New York: Plenum Press, 1988. 
[35] J.S. Wu, G.E. Apostolakis, and D. Okrent, "Uncertainty in System Analysis: probabilistic versus nonprobabilistic theories," Reliability Engineering and System Safety, vol. 30, no. 1-3, pp. 163-181, 1990.

[36] P. Baraldi, M. Compare, G. Rossetti, A. Despujols, and E. Zio, "A modelling framework to assess maintenance policy performance in electrical production plants," Maintenance Modelling and Applications, ESREDA-ESRA Project Group Report. Andrews, Berenguer and Jackson Eds., pp 263-282, 2011.

[37] P. Baraldi, A. Balestrero, M. Compare, L. Benetrix, A. Despujols, and E. Zio, "A Modeling Framework for Maintenance Optimization of Electrical Components Based on Fuzzy Logic and Effective Age," Quality and Reliability Engineering International, DOI: 10.1002/qre.1388, 2012

[38] Baraldi, P., Compare, and M., Zio, E. "Representation and propagation of the uncertainties in expert information on the parameters of degradation models for maintenance policy assessment," submitted to Applied Soft Computing.

[39] K. Sentz and S. Ferson, "Probabilistic bounding analysis in the quantification of margins and uncertainties," Reliability Engineering and System Safety, vol. 96, no. 9, pp. 1126-1136, 2011.

[40] P. Baraldi, N. Pedroni, E. Zio, E. Ferrario, A. Pasanisi, and M. Couplet, "Monte Carlo and fuzzy interval propagation of hybrid uncertainties on a risk model for the design of a flood protection dike," in European Safety and Reliability Conference 2011 (ESREL 2011), Troyes, France, 18-22 September 2011, pp 2167-2175.

[41] C. Baudrit and D. Dubois, "Practical representations of incomplete probabilistic knowledge," Computational Statistics \& Data Analysis, vol. 51, no. 1, pp. 86-108, 2006.

[42] P. Baraldi and E. Zio, "A comparison between probabilistic and Dempster-Shafer Theory approaches to Model Uncertainty Analysis in the Performance Assessment of Radioactive Waste Repositories," Risk Analysis, vol. 30, no. 7, pp. 1139-1156, 2010.

[43] E. Zio, An introduction to the basics of reliability and risk analysis, Singapore: World Scientific Publishing, 2006.

[44] G. Bojadziev and M. Bojadziev, Fuzzy Sets, Fuzzy Logic, Applications. Advances in Fuzzy Systems Applications and Theory, Vol. 5, Singapore: World Scientific, 1995.

[45] D. E. Knuth, The Art of Computer Programming, Volume 3: Sorting and Searching, Addison-Wesley, 1998. 


\section{Biographies}

Piero Baraldi (BS in nuclear engng., Politecnico di Milano, 2002; PhD in nuclear engng., Politecnico di Milano, 2006) is an assistant professor of Nuclear Engineering at the department of Energy at the Politecnico di Milano. He is the current chairman of the European Safety and Reliability Association, ESRA, Technical Committee on Fault Diagnosis. His main research efforts are currently devoted to the development of methods and techniques for system health monitoring, fault diagnosis, prognosis, and maintenance optimization. He is a co-author of 41 papers in international journals, and 48 in proceedings of international conferences; and he serves as referee of 5 international journals.

Michele Compare (BS in mechanical engng., University of Naples Federico II, 2003, PhD in nuclear engng., Politecnico di Milano, 2011) is currently a post-doc at the Politecnico di Milano. He worked as RAMS engineer, and risk manager. His main research efforts are devoted to the development of methods and techniques in support of the maintenance of complex systems.

Enrico Zio (BS in nuclear engng., Politecnico di Milano, 1991; MSc in mechanical engng., UCLA, 1995; PhD, in nuclear eng'g., Politecnico di Milano, 1995; PhD, in nuclear engng., MIT, 1998) is Director of the Chair in Complex Systems, and the Energetic Challenge of Ecole Centrale Paris and Supelec, full professor, Rector's delegate for the Alumni Association and past-Director of the Graduate School at Politecnico di Milano, adjunct professor at University of Stavanger. He is the Chairman of the European Safety and Reliability Association ESRA, member of the Korean Nuclear society and China Prognostics and Health Management society, and past-Chairman of the Italian Chapter of the IEEE Reliability Society. He is serving as Associate Editor of IEEE Transactions on Reliability, and as editorial board member in various international scientific journals. He has functioned as Scientific Chairman of three International Conferences, and as Associate General Chairman of two others. others. His research topics include analysis of the reliability, safety and security of complex systems under stationary and dynamic conditions, particularly by Monte Carlo simulation methods; and development of soft computing techniques for safety, reliability and maintenance applications, system monitoring, fault diagnosis and prognosis. He is an author or co-author of five international books, and more than 170 papers in international journals. 